\title{
CPT Category III Codes
}

National Cancer Institute

\section{Source}

National Cancer Institute. CPT Category III Codes. NCI Thesaurus. Code C155740.

Codes for tracking the use of emerging technology, services, and procedures provided by the American Medical Association as part of their Current Procedural Terminology. 